Pneumologe 2022 $\cdot 19: 63-73$

https://doi.org/10.1007/s10405-022-00434-4

Angenommen: 12. Januar 2022

Online publiziert: 11. Februar 2022

(c) The Author(s), under exclusive licence to Springer Medizin Verlag GmbH, ein Teil von Springer Nature 2022

Redaktion

S. Kluge, Hamburg

\section{Entwicklung und Fortschritt bei Atemwegsinfektionen}

\author{
Mathias W. Pletz
}

Institut für Infektionsmedizin und Krankenhaushygiene, Universitätsklinikum Jena/Friedrich Schiller Universität Jena, Jena, Deutschland

\section{Zusammenfassung}

Die Pandemie und die Ausbreitung multiresistenter Erreger zeigen eindrucksvoll, dass trotz aller medizinischer und technischer Fortschritte Infektionskrankheiten weiterhin eine globale Bedrohung darstellen. Das Auftreten neuer oder aufgrund von Resistenzen schwer zu behandelnder Infektionen wird durch die dynamische Evolution von Pathogenen bestimmt, die oft sprunghaft verlaufen kann und nicht gut vorhersagbar ist. COVID-19 hat das Verständnis von Atemwegsinfektionen verbessert und neben gegen den Erreger gerichteten Strategien den Stellenwert der Immunmodulation neu aufgezeigt. Zu den innovativen Techniken, die das Management von Atemwegsinfektionen in den nächsten Jahren verbessern werden, gehören u. a. die Erregersequenzierung, Point-of-Care-Tests und auf künstlicher Intelligenz basierende Entscheidungshilfen. Neben den viralen Atemwegsinfektionen stellen bakterielle Carbapenem-resistente Erreger (CRE) zunehmend eine therapeutische Hürde bei der nosokomialen Pneumonie dar. In den letzten Jahren wurden bei CRE wirkende Beta-Laktame zugelassen, deren Effektivität allerdings vom zugrunde liegenden Mechanismus der Carbapenem-Resistenz abhängt. Gegen Pneumokokken wird 2022 ein erweiterter 20-valenter Konjugatimpfstoff für Erwachsene zur Verfügung stehen. Gegen Influenza ist seit 2021 eine Hochdosisvakzine für ältere Erwachsene im Einsatz. Der folgende Beitrag gibt einen schlaglichtartigen Überblick über ausgewählte, maßgebliche Innovationen der letzten Jahre auf dem Gebiet der Atemwegsinfektionen.

\section{Schlüsselwörter}

Resistenzen · Immunmodulation · COVID-19 · Nosokomiale Pneumonie · Impfstoff

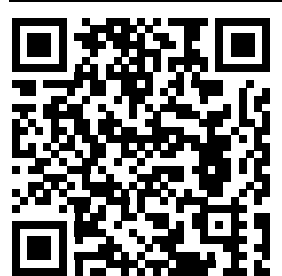

QR-Code scannen \& Beitrag online lesen
Atemwegsinfektionen - hohe Krankheitslast und viele ungelöste Fragestellungen

Die Pandemie und die Ausbreitung multiresistenter Erreger zeigen eindrucksvoll, dass trotz aller medizinischer und technischer Fortschritte Infektionskrankheiten weiterhin eine globale Bedrohung darstellen. Das Auftreten neuer oder aufgrund von Resistenzen schwer zu behandelnder Infektionen wird durch die dynamische Evolution von Pathogenen bestimmt, die oft sprunghaft verlaufen kann und nicht gut vorhersagbar ist, wie zuletzt eindrucksvoll durch das Auftreten neuer "variants of concern" von SARS-CoV-2 verdeutlicht.
Innerhalb der Infektionskrankheiten haben Atemwegsinfektionen die größte Krankheitslast. Sie gehören global zu den häufigsten Ursachen für Tod und schwere Langzeitfolgen bei Kindern und Erwachsenen. Obwohl die Krankheitslast schwer zu beziffern ist, schätzt die Weltgesundheitsorganisation (WHO), dass - präpandemisch - Infektionen der unteren Atemwegsinfektionen fast 2,6 Mio. Todesfälle pro Jahr verursachen und eine führende Todesursache bei Kindern unter 5 Jahren sind (https://www.who.int/ news-room/fact-sheets/detail/the-top10-causes-of-death). Trotz des medizinischen Fortschritts hat sich die Letalität der Pneumonie in den letzten Jahrzehnten 


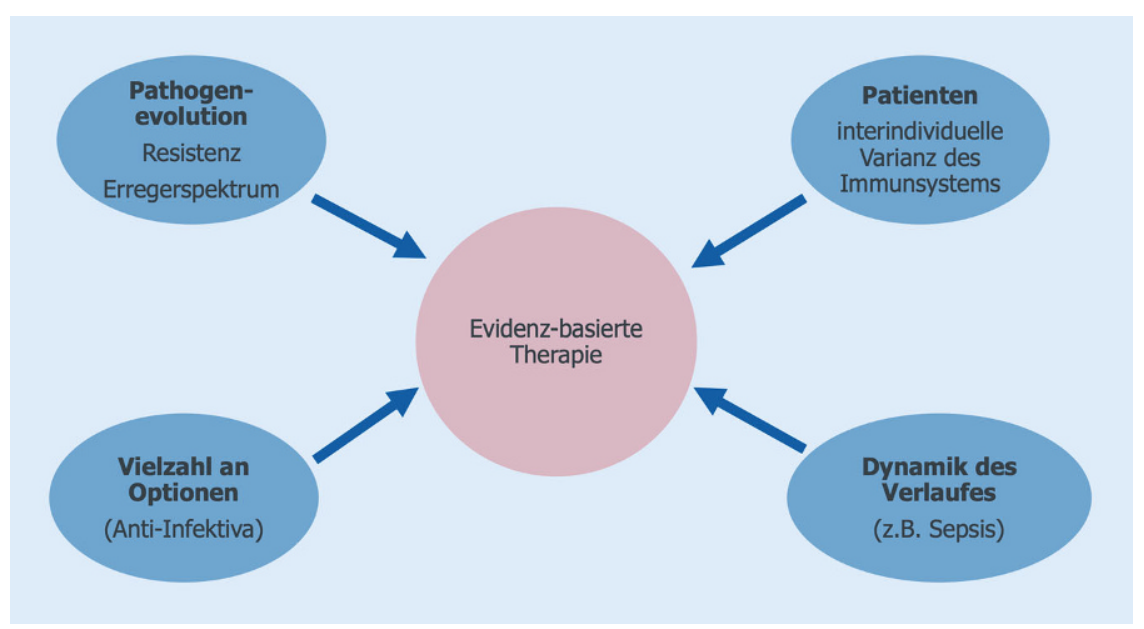

Abb. 1 \ Einflussgrößen, die die Identifikation der optimalen, evidenzbasierten Therapie im Rahmen klinischer Studien bei Atemwegsinfektionen erschweren

kaum verbessert. Dies gilt insbesondere für die reduzierte Lebenserwartung nach überlebter Pneumonie. Deren Ursache ist weiterhin nicht ganz geklärt, und wirksame Präventionskonzepte fehlen. Eine Assoziation mit dem vermehrten Auftreten postpneumonischer, kardiovaskulärer Ereignisse wurde allerdings in verschiedenen Studien beschrieben.

\section{》) Innerhalb der Infektionskrank- heiten haben Atemwegsinfektionen die größte Krankheitslast}

Industrienationen haben in den letzten Jahrzehnten zwar gelernt, die Ausbreitung fäkal-oral- (z. B. Typhus, Cholera, Brucellose) oder vektorübertragener Infektionen (z. B. Malaria, Dengue) durch technischen Fortschritt wie Kühlschränke und Klimaanlagen kontrollieren zu können. Die aktuelle Pandemie hat allerdings klar gezeigt, dass auch hoch entwickelte Industrienationen gegenüber respiratorisch übertragbaren Erregern weiterhin hoch vulnerabel sind.

Die Pandemie hat das Verständnis insbesondere für virale Atemwegsinfektionen deutlich verbessert. Im Vergleich zu nichtinfektiösen Erkrankungen gibt es bei der Erforschung von Atemwegsinfektionen einige maßgebliche Herausforderungen, die den Erkenntnisgewinn in klinischen Studien erschweren: die Heterogenität des Erregerspektrums, die hohe interindividuelle Varianz des Immunsystems und die hohe durch die verschiedenen zeitlichen
Phasen der Immunantwort bedingte intraindividuelle Varianz der Wirtsantwort. Diese in Summe hohe Variabilität hat die Beantwortung vieler Fragen zum optimalen Management der Pneumonie in klinischen Studien - insbesondere was den Stellenwert einer Immunmodulation angeht - bislang erschwert. Insofern sind Studien zu COVID-19 für den Erkenntnisgewinn besonders wertvoll, da zumindest die Heterogenität der Einflussgröße „Pathogen" wegfällt (• Abb. 1).

COVID-19-Studien belegen eindrucksvoll, dass eine gegen den viralen Erreger gerichtete Therapie nur in der frühen Phase erfolgreich ist, wohingegen in der späteren Phase der Infektion v. a. immunmodulatorische Therapien das Outcome verbessern. Diese neuen Erkenntnisse werden helfen, zukünftig auch Infektionen durch andere Erreger besser zu therapieren.

Darüber hinaus wurden in der Pandemie auch technische und strukturelle Entwicklungen vorangetrieben, die die Erforschung, das Management und die Prophylaxe von Atemwegsinfektionen zukünftig verbessern werden. Dazu gehören u. a. neue Impfstoffplattformen, die weite Verbreitung molekularer und antigenbasierter Erregertests, die Auswertung von Röntgenaufnahmen und Biomarkern mit künstlicher Intelligenz und neue Studienplattformen und -designs. Aufgrund der oben genannten Heterogenität bei Infektionskrankheiten wird der künstlichen Intelligenz Potenzial bei der optimalen individualisierten Therapie zugeschrieben.
Eine auf Maschinenlernen basierende Analyse der großen CAPNETZ-Datenbank zeigte, dass durch die individualisierte Entscheidung für oder gegen eine MakrolidBeta-Laktam-Kombination vs. einer BetaLaktam-Monotherapie bei hospitalisierten Patienten mit ambulant erworbener Pneumonie die Letalität gesenkt werden könnte [1].

\section{Erregerdiagnostik - molekulare Verfahren und Point-of-Care-Tests auf dem Vormarsch}

Die Pandemie hat zu einer nahezu ubiquitären Verfügbarkeit von Verfahren zum Nachweis von SARS-CoV-2 geführt. Hervorzuheben sind hier insbesondere in der Notaufnahme oder Praxis dezentral durchführbare Schnelltests. Der Begriff "Schnelltest" (Point-of-care-Test $[\mathrm{POC}-\mathrm{T}]=$ direkt nach Probennahme am Testort auswertbar) wird oftmals synonym mit Antigentests verwendet, da diese nur 10-15 min dauern, während die meisten PCR(Polymerasekettenreaktion)Verfahren etwa $3-4 \mathrm{~h}$ benötigen. Diese Zuordnung ist nicht mehr korrekt, da es mittlerweile spezielle PCRs gibt, die innerhalb von 20 min ein Ergebnis liefern können. Diese PCRs können in einem schuhkartongroßen, voll automatisierten Gerät durchgeführt werden, das z.B. direkt in der Notaufnahme platziert werden kann. Diese Tests sind mittlerweile auch als begrenzte Multiplex-Verfahren (COVID19, Influenza A/B, respiratorisches Synzytialvirus [RSV]) verfügbar und können v. a. in der Notaufnahme eines Krankenhauses helfen, Patienten gezielt zu isolieren und frühzeitig antiviral zu behandeln. Bereits in der schweren Influenzasaison 2017/18 haben sich solche Tests bewährt und Liegezeit und Einzelzimmerbelegungen reduziert [2].

Multiplex-Verfahren, die Viren und Bakterien nachweisen, sind bereits seit längerer Zeit im Einsatz, werden aber immer genauer und preiswerter. Eine methodische Hürde bleibt derzeit die exakte Vorhersage von Antibiotikaresistenz. Während der Nachweis einer "monogenetischen" Resistenz bei MRSA (Methicillin-resistenter Staphylococcus aureus) oder VRE (Vancomycin-resistente Enterokokken) kein Problem darstellt, ist eine umfassende, PCR-gestütz- 
Hier steht eine Anzeige.

黑 Springer 
Tab. 1 Neue Antibiotika mit (Teil-)Wirksamkeit gegen Carbapenem-resistente Erreger

\begin{tabular}{|l|l|l|}
\hline Substanz & Status & Indikation \\
\hline $\begin{array}{l}\text { Meropenem/ } \\
\text { Vaborbactam }\end{array}$ & $\begin{array}{l}\text { Zugelassen (FDA und } \\
\text { EMA) }\end{array}$ & FDA: nur cUTI, EMA: zusätzlich HAP/VAP, cIAI, BSI \\
\hline $\begin{array}{l}\text { Ceftolozan/ } \\
\text { Tazobactam }\end{array}$ & $\begin{array}{l}\text { Zugelassen (EMA und } \\
\text { FDA), verfügbar in D }\end{array}$ & cUTI, cIAI, HAP/VAP \\
\hline $\begin{array}{l}\text { Ceftazidim/ } \\
\text { Avibactam }\end{array}$ & $\begin{array}{l}\text { Zugelassen (EMA und } \\
\text { FDA), verfügbar in D }\end{array}$ & cUTI, cIAI, HAP/VAP \\
\hline Cefiderocol & $\begin{array}{l}\text { Zugelassen (EMA und } \\
\text { FDA), verfügbar in D }\end{array}$ & $\begin{array}{l}\text { EMA: aerobe GN mit „begrenzter Behandlungsmög- } \\
\text { lichkeit“ FDA: cUTI, HAP/VAP }\end{array}$ \\
\hline $\begin{array}{l}\text { Imipenem/ } \\
\text { Relebactam }\end{array}$ & $\begin{array}{l}\text { Zugelassen (EMA und } \\
\text { FDA), verfügbar in D }\end{array}$ & $\begin{array}{l}\text { FDA: CUTI, clAl, EMA: aerobe GN mit „, begrenzter Be- } \\
\text { handlungsmöglichkeit“, HAP/VAP, Bakteriämie }\end{array}$ \\
\hline $\begin{array}{l}\text { cUTI komplizierte Harnwegsinfektion, clAl komplizierte intraabdominelle Infektion, BSI Blutstrom- } \\
\text { infektion, HAP nosokomiale Pneumonie, VAP beatmungsassoziierte Pneumonie, GN gramnegative } \\
\text { Bakterien, D Deutschland, EMA European Medical Agency, FDA Food and Drug Administration }\end{array}$
\end{tabular}

te Vorhersage der Resistenz bei Gramnegativen schwierig. In methodischer Konkurrenz zu den molekularen Verfahren stehen für den Bakterien- und Resistenznachweis auch kulturbasierte, phänotypische Verfahren zur Verfügung, die nur wenige Bakterienzellen benötigen, um mittels automatisierter Mikroskopie und Vergleich mit Bilddatenbanken das Resistenzprofil zu ermitteln.

Auch die routinemäßige Sequenzierung von Erregern hat durch die Pandemie eine Beschleunigung erfahren, etwa 5-10\% der SARS-CoV-2-Isolate werden fortlaufend aus der Routine heraus sequenziert, um neue Varianten rechtzeitig zu erkennen. Bei den Sequenzierungstechniken gibt es neben dem klassischen Illumina-Verfahren, das große Geräte erfordert, das sog. Nanopore-Verfahren, das auf einem USB-Stick-großen Gerät stattfindet und sogar unter Feldbedingungen eingesetzt werden kann. Technische Visionäre gehen davon aus, dass diese sich schnell entwickelnde Methode in wenigen Jahren auch für Routinediagnostik zur Verfügung stehen wird. Verschiedene Kliniken sind bereits dazu übergegangen, aus epidemiologischen Gründen multiresistente Erreger fortlaufend zu sequenzieren, um Ausbrüche und Transmissionsketten frühzeitig zu erkennen („,Sequenzieren statt Isolieren") [3].

Es gibt mittlerweile Bestrebungen, auch Bakterien ohne vorherige Anzüchtung direkt aus Patientenmaterial zu sequenzieren, eine entsprechende klinische Studie ist bereits abgeschlossen [4]. Hier darf mit Spannung erwartet werden, wie genau die sequenzbasierte Vorhersage der Resistenz ist, denn nicht alle Resistenzgene werden exprimiert, bzw. eine Überexpression einzelner, nicht veränderter Gene kann das Resistenzspektrum erweitern. Unter- und Überexpression können aus einer Sequenzierung des Bakteriengenoms nur bedingt abgelesen werden.

\section{Neue Antiinfektiva}

\section{Antibiotika}

Neue Therapieoptionen werden v.a. gegen Carbapenem-resistente gramnegative Erreger (CRE) benötigt, die bei der nosokomialen Pneumonie eine zunehmende Rolle spielen. In Ermangelung von Alternativen galt bei diesen Erregern das nephrotoxische und nicht sonderlich wirksame Colistin als Mittel der Wahl. In den letzten Jahren wurden jedoch neue Beta-Laktame zugelassen, die deutlich besser verträglich und teilweise auch wirksamer sind als Colistin. In einer 2020 publizierten Leitlinie der Infectious Diseases Society of America werden die neuen Beta-Laktame bei Infektionen durch CRE als Mittel der Wahl gesehen, denen aufgrund besserer Wirksamkeit und Verträglichkeit gegenüber Colistin der Vorzug gegeben werden sollte [5]. In den letzten Jahren wurden nachfolgend aufgeführte neue Beta-Laktam-Antibiotika mit Wirksamkeit gegen CRE zugelassen [6].

Ceftolozan/Tazobactam wirkt gegen Erreger, deren Carbapenem-Resistenz auf einem Porinverlust beruht, dies betrifft v. a. 4MRGN(multiresistente gramnegative Bakterien mit Resistenz gegen 4 von 4 definierten Antibiotikagruppen)-Pseudomonas-aeruginosa-Isolate. Liegt eine Carba- penemase vor - der häufigste Carbapenem-Resistenz-Mechanismus bei Enterobakterien - ist es wirkungslos.

Ceftazidim/Avibactam hingegen wirkt v. a. bei Carbapenemase-Bildnern, nicht jedoch, wenn es sich um Metallo-Carbapenemasen handelt (z. B. Verona Imipenemase und New Dehli Metallobetalaktmase).

Bei Imipenem/Relebactam und $\mathrm{Me}$ ropenem/Vaborbactam handelt es sich ebenfalls um Kombinationen mit neuen Carbapenemase-Inhibitoren, die jedoch keine Wirksamkeit gegen Metallo-Carbapenemasen haben.

Hervorzuheben ist Cefiderocol [7]. Es ist der erste Vertreter einer neuen Antibiotikaklasse, der Siderophor-Cephalosporine, die gegenüber anderen Cephalosporinen einen zusätzlichen Wirkmechanismus haben: Bakterien benötigen zum Wachsen Eisenionen und konkurrieren darum mit dem Wirt. Dieser ist bemüht, während einer Infektion frei verfügbare Eisenionen zu reduzieren, Bakterien wiederum sezernieren Eisenfängermoleküle (Siderophoren), die Eisenionen in der Umgebung binden und zur Bakterienzelle zurücktransportieren, wo diese dann aktiv aufgenommen werden. Da Cefiderocol einem Siderophor ähnelt, wird es durch die Bakterien gezielt aufgenommen. Darüber hinaus ist es stabil gegen nahezu alle Carbapenemasen, einschließlich der Metallo-Carbapenemasen. Jedoch gab es in einer klinischen Studie gegenüber der Vergleichstherapie eine tendenziell erhöhte Letalität bei Infektionen mit Acinetobacter baumannii, deren Ursache noch nicht ganz verstanden ist [8].

\section{》) Der molekulare Nachweis von Resistenzdeterminanten wird auch in der Routine immer wichtiger}

Da die meisten neuen Antibiotika nur noch einen Teil der CRE - in Abhängigkeit vom zugrunde liegenden, molekularen Resistenzmechanismus - erfassen, wird zukünftig der molekulare Nachweis von Resistenzdeterminanten auch in der Routine immer wichtiger (• Tab. 1)

\section{Virulenzblocker}

Des Weiteren gibt es einen neuartigen Therapieansatz, der darauf abzielt, den erre- 
Hier steht eine Anzeige.

黑 Springer 
gerbedingten Schaden zu begrenzen, ohne einen Selektionsdruck für Resistenzentwicklung aufzubauen [9]. Es handelt sich um sog. Virulenzblocker, die bestimmte Virulenzfaktoren der Bakterien inaktivieren, ohne diese selbst abzutöten.

Eines dieser Moleküle, CAL02, bindet und inaktiviert Pneumolysin. Pneumolysin ist ein wesentlicher Virulenzfaktor von Pneumokokken, dem häufigsten bakteriellen Erreger der ambulant erworbenen Pneumonie. In einer randomisiert kontrollierten Phase-II-Studie zeigte sich, dass CAL02 - allerdings in Kombination mit einer Antibiotikatherapie - bei Patienten, die aufgrund einer Pneumokokkenpneumonie auf der Intensivstation behandelt werden mussten, zu einer schnelleren klinischen Stabilisierung führte [10]. Es bleibt abzuwarten, ob sich dieses Therapieprinzip auch bei anderen Infektionen bewährt. Einmal mehr zeigt sich jedoch, dass moderne infektionstherapeutische Konzepte ihr Potenzial nur entfalten können, wenn der zugrunde liegende Erreger und ggf. Resistenzmechanismen frühzeitig identifiziert werden. Insofern ermöglichen die oben genannten neuen diagnostischen Verfahren auch einen optimierten Einsatz neuer Substanzen.

\section{Neue Vakzinen gegen Erreger von Atemwegsinfektionen}

Die Effektivität der klassischen Influenzaspaltvakzinen ist insbesondere bei älteren Patienten aufgrund von Immunoseneszenz und bei Immunsupprimierten erniedrigt. Durch verschiedene Strategien kann die Impfantwort in dieser gefährdeten Gruppe verbessert werden. Dazu gehört neben einem Impfstoff mit Adjuvans (Immunverstärker), der schon seit einigen Jahren in Deutschland zugelassen ist, seit diesem Jahr ein Hochdosisimpfstoff, der die 4-fache Menge an Antigen erhält. In klinischen Studien unterschieden sich diese beiden Impfstoffe bei Sicherheit und Verträglichkeit nicht. Allerdings gibt es für den Hochdosisimpfstoff, der bereits seit mehreren Jahren in den USA zugelassen ist, nicht nur serologische, sondern auch klinische Daten, die eine erhöhte Wirksamkeit belegen [11]. In Studien wurden Durchbruchsinfektionen um 15-30\% gegenüber einer Impfung mit Standarddosis reduziert. Die Ständige Impfkommission (STIKO) hat berechnet, dass durch Impfung über 60-Jähriger mit dem Hochdosisimpfstoff je nach Stärke der Grippesaison bis zu etwa 250.000 symptomatische Infektionen zusätzlich verhindert werden können, und empfiehlt diesen Impfstoff für alle über 60Jährigen [12].

Gegen Pneumokokken sind für Erwachsene derzeit 2 Impfstoffe verfügbar, ein 23-valenter Polysaccharidimpfstoff und ein 13-valenter Konjugatimpfstoff [13]. Der Polysaccharidimpfstoff beinhaltet die Kapselpolysaccharide von 23 der etwa 100 bekannten Pneumokokkenserotypen. Da reine Polysacharide aber nur eine B-ZellAntwort stimulieren und kaum durch T-Zellen erkannt werden, hinterlässt er kein lang anhaltendes Gedächtnis, hat eine schlechtere Wirksamkeit bei Immunsuppression und schützt zwar zeitlich begrenzt gut vor invasiven Pneumokokkeninfektionen, definiert als Nachweis aus "sterilen“ Materialien wie Blutkultur, Liquor oder Pleurapunktat, aber nur unzureichend vor einer nichtinvasiven Pneumokokkenpneumonie mit dem Nachweis von Pneumokokken durch Antigentest oder aus respiratorischem Material. Bei der Konjugatvakzine wurden 13 Kapselpolysaccharide an ein hochimmunogenes Trägerprotein gekoppelt (nichttoxisches Diphtherietoxoid), das von T-Zellen erkannt wird. Diese T-Zellen stimulieren wiederum die B-Zellen, die auf das jeweilige Kapselpolysaccharid reagieren, und bewirken eine Antikörperreifung und Gedächtnisbildung [14].

Die Konjugatvakzine ist immunogener, hinterlässt ein Gedächtnis, wirkt besser unter Immunsuppression und verhindert - obwohl sie weniger Serotypen erfasst nach Abrechnungsdaten aus Sachsen auch mehr Pneumonien [15, 16]. Die gegenüber der Polysaccharidvakzine geringere Breite (Coverage) war bislang allerdings ein Nachteil. Allerdings hat sich die Hoffnung, dass die Impfung der Kleinkinder mit der Konjugatvakzine über Herdenprotektionseffekte zur Eradikation der 13 Serotypen auch bei Erwachsenen führt und damit die Impfung der Erwachsenen mit der Konjugatvakzine überflüssig wird, nicht erfüllt. Im Sommer 2020 wurde in den USA für Erwachsene ein Konjugatimpfstoff gegen 20 Serotypen zugelassen und bereits von der amerikanischen Impfkommission für die Impfung von Erwachsenen über 65 Jahre empfohlen. Nach Daten aus CAPNETZ unterscheidet sich die Breite dieses neuen Impfstoffes nur noch unwesentlich von der der 23-valenten Konjugatvakzine [17]. Insofern ist abzuwarten, ob dieser neue Impfstoff nach Zulassung in Deutschland die 23-valente Polysaccharidvakzine als Standardimpfung für Ältere ersetzen wird.

Neben diesen bereits oder bald verfügbaren Impfstoffen gibt es auch interessante Entwicklungen, die mittelfristig zur Verfügung stehen könnten. Dazu zählen in erster Linie Impfstoffe gegen das RespiratorySyncitial-Virus (RSV). Durch die Pandemiemaßnahmen wurde auch die globale Zirkulation anderer Atemwegsviren, u. a. RSV, stark eingeschränkt. Dies konnte u. a. durch die CAPNETZ-Studie auch in Deutschland nachgewiesen werden [18]. Infolgedessen haben insbesondere kurz vor und während der Pandemie geborene Kinder bislang kaum eine RSV-Immunität aufgebaut. Auch andere Altersgruppen sind mittlerweile wieder vulnerabler gegen RSV, da die durch eine RSV-Infektion hervorgerufene Immunität nicht dauerhaft anzuhalten scheint. Insbesondere bei Kindern verbreitet sich das Virus derzeit rapide und führt zu postpandemischen Epidemien. Daher ist damit zu rechnen, dass die klinische Prüfung für einige der über 60 RSV-VakzinKandidaten, von denen sich einige schon in Phase III befinden, eher abgeschlossen sein wird.

Ein weiterer interessanter Impfstoff ist eine "universale" Influenzavakzine, die eine jährliche Anpassung der Impfstoffe und Wiederimpfung überflüssig machen soll, indem sie sich gegen Bereiche des Virus richtet, sog. konservierte Epitope, in denen kaum Mutationen auftreten. Einige dieser Kandidaten sind in frühen klinischen Studien wegen Wirkungslosigkeit bereits gescheitert. Derzeit befinden sich 3 universale Influenzaimpfstoffe mit einem jeweils unterschiedlichen Ansatz (rekombinanter Proteinimpfstoff, Virus-likePartikel-Impfstoff und Nanopartikel-basierter Impfstoff) in Phase 3 der klinischen Entwicklung (https://ivr.cidrap.umn.edu/ universal-influenza-vaccine-technologylandscape). 
Hier steht eine Anzeige.

黑 Springer 
Tab. 2 Validierte und fortlaufend aktualisierte Literaturquellen zu COVID-19

\begin{tabular}{|l|l|l|}
\hline Thema & Herausgeber & Verweis \\
\hline $\begin{array}{l}\text { Therapie, alle Schwe- } \\
\text { regrade }\end{array}$ & WHO (Weltgesundheitsorganisation) & https://www.bmj.com/content/370/bmj.m2980 \\
\hline $\begin{array}{l}\text { Therapie, alle Schwe- } \\
\text { regrade }\end{array}$ & $\begin{array}{l}\text { Ständiger Arbeitskreis der Kompetenz- und Behandlungs- } \\
\text { zentren für Krankheiten durch hochpathogene Erreger } \\
\text { (STAKOB) }\end{array}$ & $\begin{array}{l}\text { https://www.rki.de/DE/Content/Kommissionen/Stakob/ } \\
\text { Stellungnahmen/Stellungnahme-Covid-19_Therapie_ } \\
\text { Diagnose.pdf?_blob=publicationFile }\end{array}$ \\
\hline $\begin{array}{l}\text { Therapie, alle Schwe- } \\
\text { regrade }\end{array}$ & $\begin{array}{l}\text { Fachgruppe Intensivmedizin, Infektiologie und Notfallme- } \\
\text { dizin } \\
\text { (COVRIIN) }\end{array}$ & $\begin{array}{l}\text { www.rki.de/DE/Content/InfAZ/N/Neuartiges_Coronavirus/ } \\
\text { COVRIIN_Dok/Therapieuebersicht.pdf?__blob=publicationFile }\end{array}$ \\
\hline $\begin{array}{l}\text { Infektionsprävention } \\
\text { und Management }\end{array}$ & $\begin{array}{l}\text { Arbeitsgemeinschaft der Wissenschaftlichen Medizini- } \\
\text { schen Fachgesellschaften (AWMF) bzw. verschiedene } \\
\text { Fachgesellschaften }\end{array}$ & $\begin{array}{l}\text { https://www.awmf.org/die-awmf/awmf-aktuell/aktuelle- } \\
\text { leitlinien-und-informationen-Zu-covid-19/covid-19-leitlinien. } \\
\text { html }\end{array}$ \\
\hline $\begin{array}{l}\text { Impfungen } \\
\text { htändige Impfkommission (STIKO) }\end{array}$ & $\begin{array}{l}\text { https://www.rki.de/DE/Content/Infekt/Impfen/ImpfungenAZ/ } \\
\text { COVID-19/Impfempfehlung-Zusfassung.html }\end{array}$ \\
\hline $\begin{array}{l}\text { Impfungen und } \\
\text { Antikörper }\end{array}$ & Paul-Ehrlich-Institut (PEI) & $\begin{array}{l}\text { https://www.pei.de/DE/arzneimittel/impfstoffe/covid-19/covid- } \\
19-\text { node.html }\end{array}$ \\
\hline $\begin{array}{l}\text { Diagnostik, Antigen- } \\
\text { test }\end{array}$ & $\begin{array}{l}\text { Paul-Ehrlich-Institut (PEI) } \\
\text { https://www.pei.de/SharedDocs/Downloads/DE/newsroom/ } \\
\text { dossiers/evaluierung-sensitivitaet-sars-cov-2-antigentests. } \\
\text { pdf?__blob=publicationFile\&v=69 }\end{array}$ \\
\hline
\end{tabular}

\section{Neue antivirale Medikamente}

Antivirale Medikamente sind im Vergleich zu Antibiotika schwieriger zu entwickeln, da Viren, die im Gegensatz zu Bakterien keinen eigenen Stoffwechsel mit vielen Angriffspunkten bieten, die Proteinsynthese des Wirtes für ihre Amplifikation nutzen.

Die letzte Entwicklung eines Virostatikums gegen Atemwegsviren (Oseltamivir) liegt über 20 Jahre zurück. Baloxavir marboxil (kurz Baloxavir, ehemals S-033188) ist ein in Japan entwickeltes, neues Influenzawirksames Virostatikum, das zunächst in Japan und den USA zugelassen wurde und die EMA(Europäische Arzneimittel-Agentur)-Zulassung am 07.01.2021 erhalten hat. Baloxavir hemmt nicht die Neuraminidase, sondern greift sehr früh in den Replikationszyklus von Influenza ein, indem es das sog. "cap-snatching" hemmt - das "Stehlen" einer mRNA(Messenger-Ribonukleinsäure)-Startersequenz von einer zellulären mRNA für die Virus-RNA. Baloxavir ist virostatisch deutlich wirksamer als Oseltamivir und verkürzte die mittlere Virusausscheidung in einer Studie von ca. 3 Tagen unter Oseltamivir auf 1 Tag [19]. Es hat im Gegensatz zu Oseltamivir keine eingeschränkte Wirksamkeit gegen Influenza B. Aufgrund der langen Halbwertszeit muss Baloxavir nur ein einziges Mal oral appliziert werden. Obwohl die Substanz kurzzeitig in Deutschland verfügbar war, hat sich der Hersteller entschieden, sie wieder vom Markt zu nehmen. Hintergrund war die Entscheidung des Gemeinsamen Bundesausschusses, Baloxavir gegenüber Oseltamivir nur für die Postexpositionsprophylaxe, nicht jedoch für die Therapie einen Zusatznutzen zu bescheinigen.

\section{SARS-CoV-2 und COVID-19}

Die Entwicklung neuer diagnostischer, therapeutischer und präventiver Konzepte gegen COVID-19 ist von einer hohen Dynamik gekennzeichnet und so umfangreich, dass eine adäquate Darstellung den Umfang des Beitrags sprengen würde. Zu COVID19 sind mittlerweile über 200.000 Peer-reviewed-Artikel in PubMed erschienen. Es ist außerdem zu erwarten, dass bei Druck dieses Beitrags bereits weitere Substanzen und Vakzinen zugelassen sind. Daher sollen im Folgenden nur die Grundzüge und wesentlichen Erkenntnisse der entsprechenden Entwicklungen nachgezeichnet werden. Bezüglich einer fortlaufenden, ständig aktualisierten Übersicht zu Therapeutika, Impfstoffen und Diagnostika wird auf die Institutionen und Links in 0 Tab. 2 verwiesen.

\section{Antivirale Medikamente}

Zu Beginn der Pandemie wurden verschiedene bereits zugelassene Substanzen in klinischen Studien auf eine Wirksamkeit gegen SARS-CoV-2 getestet (,repurposing ${ }^{\prime \prime}$ ). Während kleine Pilotstudien initial oft eine gewisse Wirksamkeit vermuten ließen, konnte diese in großen multizentrischen Studien für die meisten getesteten Substanzen nicht belegt werden. Azithromycin, Lopinavir/Ritonavir, Ivermectin und Interferon zeigten in den großen Plattform-Studien (REMAP-CAP, SOLIDARITY, ATTAC) keine Wirkung [20]. Auch bei Remdesivir, ein gegen Ebola und Hepatitis $C$ entwickeltes Breitspektrumvirostatikum, gab es zwar in der Phase-III-Studie des Herstellers eine Wirksamkeit, die in der WHO-Solidarity-Studie aber nicht bestätigt werden konnte [21].

\section{॥ In der späten Phase zum} Zeitpunkt der Hospitalisierung kommen Virostatika oftmals zu spät

Die enttäuschende Effektivität dieser antiviralen Medikamente bei hospitalisierten Patienten erklärt sich teilweise aus der Pathophysiologie und dem Verlauf der Infektion. In Analogie zu Influenza spielt die Virusreplikation zwar eine entscheidende Rolle in der Frühphase der Infektion, in der späten Phase zum Zeitpunkt der Hospitalisierung dominiert das Problem der überschießenden Inflammation durch die fehlregulierte Wirtsantwort [22]. In dieser Phase kommen Virostatika oftmals zu spät. Der enge Korridor der Empfehlung für den Einsatz von Remdesivir illustriert dieses Problem - Patienten, die mit hoher Wahrscheinlichkeit einen schweren Verlauf nehmen und bereits Low-flow-Sauerstoffgabe benötigen, aber noch nicht in die 
Hier steht eine Anzeige.

黑 Springer 
Phase der überschießenden Wirtsantwort eingetreten sind.

Remdesivir war aufgrund seiner fehlenden oralen Verfügbarkeit keine Option für den ambulanten Bereich. Hier stehen mit Paxlovid (Pfizer), einem speziell gegen SARS-CoV-2 entwickelten Proteaseinhibitor und Molnupirvir (Merck) 2 oral applizierbare Medikamente zur Verfügung, die bei ambulanten Patienten mit Risikoprofil das Risiko der Krankenhauseinweisung signifikant reduzieren können [23, 24]. Bei Molnupiravir handelt es sich um ein $\mathrm{Nu}$ kleosidanalogon, das die Mutationsrate bei der Virusreplikation so stark erhöht, dass keine replikationsfähigen Viren mehr entstehen [25].

Eine weitere Option für frühe Krankheitsphasen sind monoklonale Antikörper, die auch zur passiven Vakzinierung, zur Postexpositionsprophylaxe und zur präemptiven Therapie (positiver Virusnachweis bei - noch - fehlender Symptomatik) eingesetzt werden können. Die Wirksamkeit der monoklonalen Antikörper kann jedoch durch das Auftreten neuer Varianten deutlich abgeschwächt werden.

\section{Immunmodulatoren}

Der bislang größte Effekt bezüglich Reduktion der COVID-19-Letalität bei intensivpflichtigen Patienten konnte für Dexamethason gezeigt werden [26]. Dies war in gewisser Weise überraschend, da bei Influenza wiederholt gezeigt wurde, dass Steroide die Sterblichkeit eher erhöhen [27]. Aber auch bei COVID-19 zeigte sich der positive Effekt der Steroide bei Patienten in der späten Phase der Erkrankung, während es bei Patienten in der Frühphase einen nachteiligen Trend gab.

Weitere immunmodulierende Substanzen, deren Effektivität in randomisierten Studien belegt werden konnte und die in der neuen S2K-Leitlinie zu hospitalisierten Patienten mit COVID-19 genannt werden, sind Interleukin-6-Blocker und Januskinaseinhibitoren [28]. Kürzlich hat auch Anakinra (Interleukin-1-Blockade) eine Indikationserweiterung für hospitalisierte Patienten mit COVID-19 erhalten [29].

Neben der Immunmodulation kommt der Antikoagulation, die der Bildung von Mikro- und Makrothromben auf dem Boden der SARS-COV-2-typischen Endothe- liitis [30] entgegenwirkt, eine wesentliche Bedeutung zu. Überraschenderweise zeigte die große REMAP-CAP-Studie, dass bei Patienten auf der Intensivstation eine prophylaktische Antikoagulation der therapeutischen wegen des geringeren Risikos von Blutungskomplikationen überlegen ist [31].

\section{Vakzinen}

Im Rahmen der Pandemie wurden mit den mRNA- und Vektor-Impfstoffen 2 neue Vakzineplattformen in die breite klinische Anwendung gebracht. Für die leicht adaptierbaren mRNA-Impfstoffe wird aktuell auch eine Anwendung zur therapeutischen Vakzinierung bei fortgeschrittenen Tumorerkrankungen untersucht (u.a. Melanom und Kolonkarzinom). Insgesamt hat die Pandemie die Impfstoffforschung stark vorangebracht und das Verständnis der interindividuell stark verschiedenen Immunantwort erweitert. Dadurch wurde aber auch Bewährtes, z. B. die Definition eines protektiven Titers, infrage gestellt.

\section{Fazit für die Praxis}

- Die Pandemie und die Ausbreitung multiresistenter Erreger zeigen, dass trotz aller medizinischer und technischer Fortschritte Infektionskrankheiten weiterhin eine globale Bedrohung darstellen.

- COVID-19 hat das Verständnis von Atemwegsinfektionen verbessert und neben gegen den Erreger gerichteten Strategien den Stellenwert der Immunmodulation neu aufgezeigt.

- Zu den innovativen Techniken, die das Management von Atemwegsinfektionen in den nächsten Jahren verbessern werden, gehören u.a. die Erregersequenzierung, Point-of-Care-Tests und auf künstlicher Intelligenz basierende Entscheidungshilfen.

- Die Effektivität der bei bakteriellen Carbapenem-resistenten Erregern (CRE) wirkenden Beta-Laktame hängt vom zugrunde liegenden Mechanismus der Carbapenem-Resistenz ab.

- Gegen Pneumokokken wird 2022 ein erweiterter 20-valenter Konjugatimpfstoff für Erwachsene zur Verfügung stehen.

- Gegen Influenza ist seit 2021 eine Hochdosisvakzine für ältere Erwachsene im Einsatz.

\section{Korrespondenzadresse}

\section{Mathias W. Pletz}

Institut für Infektionsmedizin und Krankenhaushygiene, Universitätsklinikum Jena/Friedrich Schiller Universität Jena

07740 Jena, Deutschland

Mathias.Pletz@med.uni-jena.de

\section{Einhaltung ethischer Richtlinien}

Interessenkonflikt. M.W. Pletz gibt folgende Interessenkonflikte an: Referentenhonorare von Bayer, GSK, Chiesi, MSD, Novartis, Pfizer, Roche und ThermoFisher; Forschungsunterstützung von DFG, BMBF und Pfizer; Teilnahme an Advisory Boards (Honorar an Arbeitgeber) von GSK, Chiesi, MSD, Novartis, Pfizer und Roche.

Für diesen Beitrag wurden vom Autor keine Studien an Menschen oder Tieren durchgeführt. Für die aufgeführten Studien gelten die jeweils dort angegebenen ethischen Richtlinien.

\section{Literatur}

1. König R, Cao X, Oswald M, Forstner C, Rohde G, Rupp J et al (2019) Macrolide combination therapy for patients hospitalised with communityacquired pneumonia? An individualised approach supported by machine learning. Eur Respir J 54(6):1900824

2. Youngs J, Marshall B, Farragher M, Whitney L, Glass S, Pope C et al (2019) Implementation of influenza point-of-care testing and patient cohorting during a high-incidence season: a retrospective analysis of impact on infection prevention and control and clinical outcomes. JHosp Infect 101:276-284

3. Mellmann A, Bletz S, Böking T, Kipp F, Becker K Schultes $A$ et al (2016) Real-time genome sequencing of resistant bacteria provides precision infection control in an institutional setting. J Clin Microbiol 54:2874-2881

4. Brenner T, TIFOnet Critical Care Trials Group. (2020) "Next Generation Sequencing" zur Diagnostik der Bakteriämie bei Sepsis - Next GeneSiS-Trial. Anaesthesist 69:593-595

5. Tamma PD, Aitken SL, Bonomo RA, Mathers AJ van Duin D, Clancy CJ (2021) Infectious disease society of america guidance on the treatment of extended-spectrum $\beta$-lactamase producing enterobacterales (ESBL-E), carbapenem-resistant enterobacterales (CRE), and pseudomonas aeruginosa with difficult-to-treat resistance (DTR-P. aeruginosa). Clin Infect Dis 72:1109-1116

6. Hites M (2021) Minireview on novel anti-infectious treatment options and optimized drug regimens for sepsis. Front Med (Lausanne) 8:640740

7. Yao J, Wang J, Chen M, Cefiderocol CY (2021) An overview of its in-vitro and in-vivo activity and underlying resistant mechanisms. Front Med (Lausanne) 8:741940

8. Bassetti M, Echols R, Matsunaga $Y$, Ariyasu M, Doi Y, Ferrer R et al (2021) Efficacy and safety of cefiderocol or best available therapy for the treatment of serious infections caused by carbapenem resistant Gram-negative bacteria (CREDIBLE-CR): a randomised, open-label, multicentre, pathogenfocused, descriptive, phase 3 trial. Lancet Infect Dis 
21(2):226-240. https://doi.org/10.1016/S14733099(20)30796-9

9. Pletz MW, Bauer M, Brakhage AA (2019) One step closer to precision medicine for infectious diseases. Lancet InfectDis 19:564-565

10. Laterre P-F, Colin G, Dequin P-F, Dugernier T, Boulain T, Azeredo da Silveira $S$ et al (2019) CAL02, a novel antitoxin liposomal agent, in severe pneumococcal pneumonia: a first-in-human, double-blind, placebo-controlled, randomised trial. Lancet Infect Dis 19:620-630

11. Lee JKH, Lam GKL, Shin T, Kim J, Krishnan A, Greenberg DP et al (2018) Efficacy and effectiveness of high-dose versus standard-dose influenza vaccination for older adults: a systematic review and meta-analysis. Expert Rev Vaccines 17:435-443

12. STIKO (2021) Beschluss und Wissenschaftliche Begründungder Ständigen Impfkommission (STIKO)für die Aktualisierung der InfluenzaImpfempfehlungfür Personen im Alter von $\geq 60$ Jahren, $\mathrm{S} 34$

13. Pletz MW, Bahrs C (2021) Pneumococcal vaccination. Internist (Berl) 62:807-815

14. Pletz MW, Maus U, Krug N, Welte T, Lode H (2008) Pneumococcal vaccines: mechanism of action, impact on epidemiology and adaption of the species. Int J Antimicrob Agents 32:199-206

15. Kolditz M, Schmitt J, Pletz MW, Tesch F (2018) Impact of pneumococcal polysaccharide vaccine on incidence and mortality after pneumonia in adults aged $\geq 60$ years - a population-based retrospective cohort study. Clin Microbiol Infect 24(5):500-504. https://doi.org/10.1016/j.cmi. 2017.08.010

16. Kolditz M, Schmitt J, Pletz MW, Tesch F (2018) Impact of the 13-valent pneumococcal conjugate vaccine on incidence of all-cause pneumonia in adults aged $\geq 60$ years - a populationbased retrospective cohort study. Clin Infect Dis 68:2117-2119

17. Bahrs C, Kesselmeier M, Kolditz M, Ewig S, Rohde G, Barten-Neiner Getal (2021) A longitudinal analysis of pneumococcal vaccine serotypes in pneumonia patients in Germany. Eur Respir J. https://doi.org/ 10.1183/13993003.02432-2021

18. Dähne T, Bauer W, Essig A, Schaaf B, Spinner CD, Pletz MWetal (2021) The impact of the SARS-CoV-2 pandemic on the prevalence of respiratory tract pathogens in patients with community-acquired pneumonia in Germany. Emerg Microbes Infect 10:1515-1518

19. Hayden FG, Sugaya N, Hirotsu N, Lee N, de Jong MD, Hurt AC et al (2018) Baloxavir marboxil for uncomplicated influenza in adults and adolescents. N Engl J Med 379:913-923

20. WHO Solidarity Trial Consortium, Pan H, Peto R, Henao-Restrepo A-M, Preziosi M-P, Sathiyamoorthy $V$ et al (2021) Repurposed antiviral drugs for Covid-19 - interim WHO solidarity trial results. NEngl J Med 384:497-511

21. Okoli GN, Rabbani R, Copstein L, Al-Juboori A, Askin N, Abou-Setta AM (2021) Remdesivir for coronavirus disease 2019 (COVID-19): a systematic review with meta-analysis and trial sequential analysis of randomized controlled trials. Infect Dis (Lond) 53:691-699

22. Karakike E, Giamarellos-Bourboulis EJ, Kyprianou M, Fleischmann-StruzekC,Pletz MW, Netea MG et al (2021) Coronavirus disease 2019 as cause of viral sepsis: a systematic review and meta-analysis. Crit Care Med 49:2042-2057

23. Mahase E (2021) Covid-19: Pfizer's paxlovid is $89 \%$ effective in patients at risk of serious illness, company reports. BMJ 375:n2713

\section{Development and progress in respiratory tract infections}

The pandemic and the spread of multidrug-resistant pathogens impressively demonstrate that despite all medical and technical progress, infectious diseases still represent a global threat. The occurrence of new or difficult to treat infections due to resistance is determined by the dynamic evolution of pathogens, which can often have an erratic course and is not easily predictable. Coronavirus disease 2019 (COVID-19) has improved the understanding of airway infections and in addition to strategies targeted against the pathogen, has again demonstrated the importance of immunomodulation. Pathogen sequencing, point of care testing and decision aids based on artificial intelligence are some of the innovative techniques which will improve the management of airway infections in the coming years. In addition to viral airway infections, bacterial carbapenem-resistant pathogens (CRE) increasingly represent a therapeutic barrier in cases of nosocomial pneumonia. In recent years approval was given to beta lactams that are effective against CRE; however, their effectiveness is dependent on the underlying mechanism of resistance to carbapenem. An extended 20-valent pneumococcal conjugate vaccine will become available in 2022 for adults. A high-dose vaccine against influenza has been in use since 2021 for older adults. This article provides a highlighted overview of selected significant innovations in recent years in the field of airway infections.

\section{Keywords}

Resistance $\cdot$ Immunomodulation · COVID-19 $\cdot$ Nosocomial pneumonia $\cdot$ Vaccine

24. Fischer WA, Eron JJ, Holman W, Cohen MS, Fang L, Szewczyk LJ et al (2021) A phase 2a clinical trial of molnupiravir in patients with COVID-19 shows accelerated SARS-coV-2 RNA clearance and elimination of infectious virus. Sci Transl Med. https://doi.org/10.1126/scitranslmed.abl7430

25. Pourkarim F, Pourtaghi-Anvarian S, Rezaee $\mathrm{H}$ (2022) Molnupiravir: a new candidate for COVID-19 treatment. Pharmacol Res Perspect 10:e909

26. RECOVERY Collaborative Group, Horby P, Lim WS, Emberson JR, Mafham M, Bell JL et al (2021) Dexamethasone in hospitalized patients with Covid-19. N Engl J Med 384:693-704

27. Lansbury L, Rodrigo C, Leonardi-Bee J, NguyenVan-Tam J, Lim WS (2019) Corticosteroids as adjunctive therapy in the treatment of influenza. Cochrane Database Syst Rev 2:CD10406

28. Kluge S, Janssens U, Welte T, Weber S (2020) S2kLeitlinie-Empfehlungen zur stationären Therapie von Patienten mitCOVID-19

29. Kyriazopoulou E, Poulakou G, Milionis H, Metallidis S, Adamis G, Tsiakos K et al (2021) Early treatment of COVID-19 with anakinra guided by soluble urokinase plasminogen receptor plasma levels: a double-blind, randomized controlled phase 3 trial. Nat Med 27:1752-1760

30. Ackermann M, Verleden SE, Kuehnel M, Haverich A, Welte T, Laenger $\mathrm{F}$ et al (2020) Pulmonary vascular endothelialitis, thrombosis, and angiogenesis in Covid-19.NEngl JMed 383:120-128

31. REMAP-CAP Investigators, ACTIV-4a Investigators, ATTACC Investigators, Goligher EC, Bradbury CA, McVerry BJ et al (2021) Therapeutic anticoagulation with heparin in critically ill patients with Covid-19. N Engl J Med 385:777-789 